\title{
Bone morphogenetic protein signaling in rotator cuff muscle atrophy and fatty infiltration
}

\author{
Xuhui Liu'1,2 \\ Sunil Joshi ${ }^{1}$ \\ Bharat Ravishankar1,2 \\ Dominique Laron ${ }^{2}$ \\ Hubert T. Kim ${ }^{1,2}$ \\ Brian T. Feeley ${ }^{1,2}$
}

1 San Francisco Veterans Affairs Medical Center, Department of Veterans Affairs, San Francisco, USA

2 Department of Orthopaedic Surgery, University of California, San Francisco, USA

Corresponding author:

Brian T. Feeley

Department of Orthopaedic Surgery, University of California

1500 Owens Ave, Box 3004

94158 San Francisco, CA, USA

E-mail: feeleyb@orthosurg.ucsf.edu

\section{Summary}

Background: reduced mass (atrophy) and increased fat content (fatty infiltration) of rotator cuff muscles are common complications of large or massive rotator cuff (RC) tears, and are believed to be irreversible even after tendon repairs. Clinically, both muscle atrophy and fatty infiltration are important factors contributing to poor functional outcomes after tendon repairs. The molecular mechanism of RC muscle atrophy and FI remains undefined. In this study, we investigated the role of bone morphogenetic proteins (BMP) signaling in RC muscle atrophy and fatty infiltration using a rat model.

Methods: unilateral massive RC tears was induced in adult rats. RC muscles were harvested at 2 and 6 weeks after injury for BMP signaling analysis. In a separate experiment, BMP inhibitor (LDN-193189) was injected to rats through daily intraperitoneal injection. RC muscles from rats in the treated and control groups were harvested at 6 weeks after injury for biochemistry and histology analysis.

Results: we found significantly increased BMP-14 and BMP-7 expression in rotator cuff muscles after RCT. Inhibiting BMP signaling resulted in increased muscle atrophy and reduced fatty infiltration in rotator cuff muscle after $\mathrm{RC}$ tears.
Conclusion: this result suggests that BMP signaling inhibits RC muscle atrophy but promotes fatty infiltration.

KEY WORDS: atrophy, BMP, fatty infiltration, muscle, rotator cuff tear.

\section{Introduction}

Rotator cuff tears (RCTs) are the most common upper extremity injury seen by orthopaedic surgeons and primary care physicians, and the incidence of rotator cuff surgery continues to rise ${ }^{1}$. The outcome of repairing small RCTs is good, but there has been limited success in the surgical treatment of massive RCTs. Clinically, both muscle atrophy and fatty infiltration, have been correlated with poor functional outcome following large RCT repair ${ }^{2}$. The natural history of muscle atrophy and fatty infiltration in the rotator cuff has been well studied in the last few years. In a series of retrospective reviews, Melis et al. performed a review of 1688 patients with rotator cuff tears to determine the natural history of fatty infiltration and atrophy $^{3-5}$. Fatty infiltration was related to increased patient age, larger tears, and delay between onset and diagnosis. In a recent study, Chung et al. demonstrated that higher fatty infiltration of the infraspinatus muscle was the single most important factor negatively affecting cuff healing after arthroscopic repair of massive rotator cuff tears ${ }^{6}$.

The underlying molecular mechanisms that lead to the development of rotator cuff muscle atrophy and fatty infiltration remain undefined. Thus, there is no effective treatment for rotator cuff muscle pathology at this time. Bone morphogenetic proteins (BMPs) are a subgroup of the transforming growth factor beta (TGF $\beta$ ) super family and have been extensively studied in their role in bone formation. Although some studies suggest BMP signaling regulates embryonic muscle development, current research provides limited insight into whether BMP signaling operates in post mitotic myofibers to regulate skeletal muscle phenotype. BMPs engage serine/threonine kinase receptors that phosphorylate Smad1/5/8, which promotes binding of Smad4 and subsequent nuclear translocation which governs gene expression in a cell- and context- dependent manner. Winbanks et al. recently reported that BMP pathway is a positive regulator of muscle mass $^{7}$. Similarly, Sartori et al. determined that inhibition of BMP signaling causes muscle atrophy through 
the regulation of the ubiquity in ligase family ${ }^{8}$. However, the role of BMP signaling pathway in rotator cuff muscle pathology has not been identified.

In this study, we investigated the role of BMP signaling in rotator cuff muscle atrophy and fatty infiltration using a rat RCT model. We hypothesize that BMP signaling contributes to rotator cuff muscle atrophy and fatty infiltration after RCT.

\section{Materials and methods}

Our study meets the ethical standards of the Journal ${ }^{9}$.

\section{Animal Surgery and tissue process}

Adult female Sprague Dawley rats (Charles River Laboratories Inc., Wilmington, MA) weighed 200-250g were used for surgeries. A combined supraspinatus and infraspinatus tendon transection and SSN transection surgery $(T T+D N)$ was performed on the right shoulder as previously described in order to simulate a massive RCT accompanied with nerve injury ${ }^{9}$. Sham surgery was performed on the contralateral side to serve as an internal control. All procedures were approved by San Francisco Veterans Affairs Medical Center (SFVAMC) Institutional Animal Care and Use Committee (IACUC). Rats were sacrificed 2 weeks or 6 weeks after surgery ( $\mathrm{N}=6$ at each time point). Supraspinatus muscles from both surgical and sham sides were harvested and the remaining tendon and scar tissue were removed at the muscle/tendon junction. The wet weight of supraspinatus muscles of both surgery and sham sides were measured immediately for analysis of muscle atrophy ${ }^{10}$. Muscles were subsequently divided in half for protein and RNA extraction ( $\mathrm{N}=6$ in each group) by homogenizing in T-PER solution (Pierce Biotechnology Inc. Rockford, IL.) with a protease inhibitor cocktail (SigmaAldrich Inc. St Louis, MO) or Trizol® reagent (Invitrogen Inc. Carlsbad, CA) ${ }^{11,12 .}$

\section{BMP inhibitor treatment}

Twenty-four rats were used for inhibitor experiments. Rats were randomly divided into treatment and control groups ( $\mathrm{N}=12$ in each group) (Tab. 1). A combined supraspinatus and infraspinatus tendon transection and SSN transection surgery (TT+DN) described above was performed on the right shoulder. Sham surgery was performed on the contralateral side. Rats in the treatment group received daily intraperitoneal injection of LDN-193189 (Sellect Chem Inc. Houston, TX) at a dose of $5 \mathrm{mg} / \mathrm{kg}$. Rats in the control group received daily intraperitoneal injection of vehicle $(0.1 \%$ DMSO in saline). Rats were sacrificed at 6 weeks after surgery. Supraspinatus muscles from both surgical and sham sides were harvested and weighted immediately. Muscles from six rats in each group were used for histology analysis. Muscles from the remaining rats were divided for protein and RNA extraction, respectively.

\section{Histologic analysis}

Muscle samples for histology analysis were flash frozen and were sectioned at $-20^{\circ} \mathrm{C}$ at a thickness of $10 \mu \mathrm{m}$. Only sections at the belly of the muscles were used for histological analysis. Oil-red $O$ staining was performed to identify fatty infiltration in muscles. In brief, frozen sections were fixed in $3.7 \%$ formaldehyde for one hour and then stained with a working Oil-Red-O solution 30 minutes. Slides were then mounted in a $10 \%$ glycerol solution. Pictures of the samples were taken using an Apotome fluorescence microscope (Zeiss Apotome 2 with Axiovert 200M, Carl Zeiss Inc.Thornwood, NY) with Axio Cam MRm camero (Zeiss Inc.). The area of fat (stained in red with Oil-red $\mathrm{O}$ ) on each section was measured using Image $\mathrm{J}(\mathrm{NIH})$ and were calculated as the percentages of fat area over the cross-sectional area of the muscle section. All slides were reviewed by two blinded researchers.

\section{Western-blot analysis}

After determining protein concentration with a bicinchoninic acid (BCA) Protein Assay Kit (Thermo Scientific Inc. Rockford, IL), $40 \mu \mathrm{g}$ of protein was loaded on $10 \%$ NUPAGE Bis-Tris gels and transferred to Immobilon-FL PVDF membranes (Millipore Inc., Billerica, MA) that were blocked and incubated in primary

Table 1. Experimental Set-up \& Animal Number.

\begin{tabular}{lll}
\hline & No BMP Inhibition: & Sham \\
\hline 2 \& 6 weeks following RCTs (N=6/time-point) & TT+DN & Western Blotting \\
& qRT-PCR & $\begin{array}{l}\text { Western Blotting } \\
\text { qRT-PCR }\end{array}$ \\
\hline 6 weeks of BMP Inhibitor Administration: & Vehicle Group \\
\hline Biochemical Analysis (western blotting \& qRT-PCR) & $\mathrm{N}=6$ & $\mathbf{N}=\mathbf{1 2})$ \\
\hline Histological Analysis & $\mathrm{N}=6$ & $\mathrm{~N}=6$ \\
\hline
\end{tabular}


and secondary antibodies. The following rabbit-antirat primary antibodies were used at the given dilutions: phospho-SMAD-1/5/8 (1:250) (Cell Signaling Technology Inc., Danvers, MA) and sterol regulatory element-binding protein 1 (SREBP-1) (1:200) (Santa Cruz Biotechnology Inc. Santa Cruz, CA). A fluorescent IRDye $800 \mathrm{CW}$ Goat anti-Rabbit IgG secondary antibody (LI-COR Biosciences, Lincoln, NE) was used at a dilution of 1:10,000. Blots were imaged using the Odyssey Infrared Imaging System (LI-COR Biosciences) and quantified using Image J Software (NIH).

\section{Quantitative Reverse Transcription Polymerase Chain Reaction (qRT-PCR)}

Total RNA was isolated from muscle with Trizol reagent as previously described. One microgram of total RNA from each sample was used to synthesize cDNA using a Transcriptor First Strand cDNA Synthesis Kit (Roche Applied Bioscience Inc., Indianapolis, IN.). Quantitative PCR was performed to quantify the expression of BMP-1,2,3,4,5,6,7,13 and 14, MuRF-1, MAfbx and FOXO3 in our muscle samples using a Light Cycler 480 SYBR Green I Master kit (Roche Applied Bioscience Inc.). Amplification reactions were performed with 40 cycles of $\left(95^{\circ} \mathrm{C}\right.$ for $10 \mathrm{~min} ; 58^{\circ} \mathrm{C}$ for $30 \mathrm{~s}$; and $72^{\circ} \mathrm{C}$ for $1 \mathrm{~min}$ ). Primers was synthesized by Elim Biopharm Inc. (Hayward, CA) and sequence of each primer was listed in Table 2. Each gene was run in triplicates for each sample. Ct values was calculated as the mean of triplicates and normalized to that of GAPDH. Fold change in mRNA expression was calculated by using $\Delta \Delta \mathrm{Ct}$ method as described previously ${ }^{13}$. In the non-inhibitor treatment experiment, the gene expression level in muscles from the surgery side was normalized to those from the sham side in each animal. In BMP-inhibitor treat- ment experiment, the gene expression level in muscles from the surgery side in the treated group was compared to that in muscles from the surgery side in the control groups.

\section{Statistical analysis}

A paired T-test was used to compare differences between surgery and sham side for the animals without inhibitor/vehicle treatment. For inhibitor experiment, an unpaired T-test was used to compare the difference between the treated and control groups. Significance was defined as a $p$ value of less than 0.05. Data is presented as the mean \pm standard error.

\section{Results}

Increased gene expression BMP-7 and BMP-14 in supraspinatus muscle after RCT

Real-time RT-PCR demonstrated that among the BMP family panel tested, the expression of BMP-14 significantly increased at 2 weeks after surgery (compared to sham). At six weeks after surgery, the expression level of BMP-14 was consistently up-regulated. The expression of BMP-7 was also significantly up-regulated at 6 weeks after surgery. There was no significant change in expression in the remainder of the BMP panel we tested (Fig. 1).

\section{Increased canonical BMP pathway in supraspina- tus muscle after RCT}

Western-blot analysis showed that the level of phosphorylated (active) Smad1/5/8, the immediate downstream effector of the canonical BMP pathway was

Table 2. Primer Sequences.

\begin{tabular}{|c|c|c|}
\hline Gene & Forward (5’ $\left.\rightarrow 3^{\prime}\right)$ & Reverse (5’ $\rightarrow 3$ ') \\
\hline BMP-1 & CAATGGCTACTCTGCCCACA & CAACCCAATTGCTGCTGCTT \\
\hline BMP-2 & GCAGCTTCCATCACGAAGA & GATGTGAGAAACTCGTCACTGG \\
\hline BMP-3 & TGGGGCCGAGTATCAGTACA & ACTTTAAGGTACCTGCGGGC \\
\hline BMP-4 & TTCCGTAGTGCCATTCCGAG & ACGACCATCAGCATTCGGTT \\
\hline BMP-5 & ATCGCTGTGCTCCACTTCA & ATGTCAACGCTCTCACCCAG \\
\hline BMP-6 & AGCAACAATCGCAACAGACG & GATCCAGCATGAAGAGCGGA \\
\hline BMP-7 & AGCTTCGTCAACCTAGTGGAAC & CTGGAGCACCTGATAGACTGTG \\
\hline BMP-13 & AAGACTTACTCCATTGCCGAGA & TCGTCCAGTCCTCTGTCTACAA \\
\hline BMP-14 & ATGCTGACAGAAAGGGAGGTAA & GCACTGATGTCAAACACGTACC \\
\hline MuRF-1 & ACCTGCTGGTGGAAAACATC & CTTCGTGTTCCTTGCACATC \\
\hline MAFbx & GCAAACACTGCCACATTCTCTC & CTTGAGGGGAAAGTGAGACG \\
\hline FOXO3 & ACTTCAAGGATAAGGGCGACAGCA & СTTCATTCTGAACGCGCATGAAGC \\
\hline GAPDH & CACCATCTTCCAGGAGCGAG & ССTTCTCCATGGTGGTGAAGAC \\
\hline
\end{tabular}


significantly up-regulated at both 2 and 6 weeks after surgery compared to sham (Fig. 2).

\section{LDN 193189 reduced phosphorylated Smad level in supraspinatus muscle after RCT}

After 6 weeks of treatment of LDN 193189, a potent inhibitor of BMP signaling by adhering to the ALK-1 receptor, the level of phosphorylated (active) Smad1/5/8 significantly decreased in supraspinatus muscles at the surgery side (Fig. 3). This suggested that daily injection of LDN 193189 effectively inhibited the canonical BMP signaling in rotator cuff muscle after RCT.

Inhibiting BMP signaling leads to increased supraspinatus muscle weight loss

Six weeks of LDN 193189 treatment, there was not significantly difference on body weight between the animals in treated group $(282 \pm 16.9 \mathrm{~g})$ and those in the vehicle treated control group $(273 \pm 15.4 \mathrm{~g})$ $(p=0.62)$. However, there was $12.5 \%$ of reduction of normalized supraspinatus muscle weight (weight of supraspinatus/body weight) in LDN-193189 treated group $(0.049 \pm 0.0031 \mathrm{~g} / \mathrm{kg})$ compared to that of vehicle-treated control group $(0.056 \pm 0.0029 \mathrm{~g} / \mathrm{kg})$

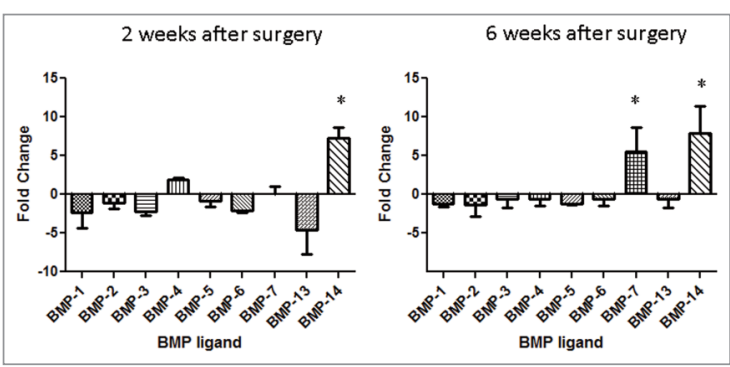

Figure 1. Fold change of BMP1-7, 13 and 14 in supraspinatus muscles at 2 and 6 weeks after surgery. $\left({ }^{*}, p<0.05\right.$ compared to sham side).

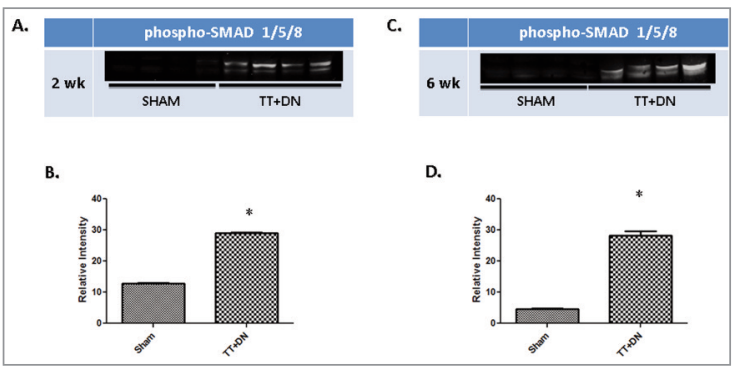

Figure 2. Typical images and band intensity quantification of Western-blot of phosphor-Smad1/5/8 in supraspinatus muscles at 2 and 6 weeks after surgery. $\left({ }^{*}, p<0.05\right.$ compared to sham side). $(p=0.005)$ (Fig. 4). LDN 193189 also significantly increased the expression of MuRF-1 $(p=0.013)$ and MAFbx $(p=0.017)$, two muscle atrophy specific genes that activate the ubiquity in ligase protein degradation pathway in supraspinatus muscles after RCT (Fig. 5).

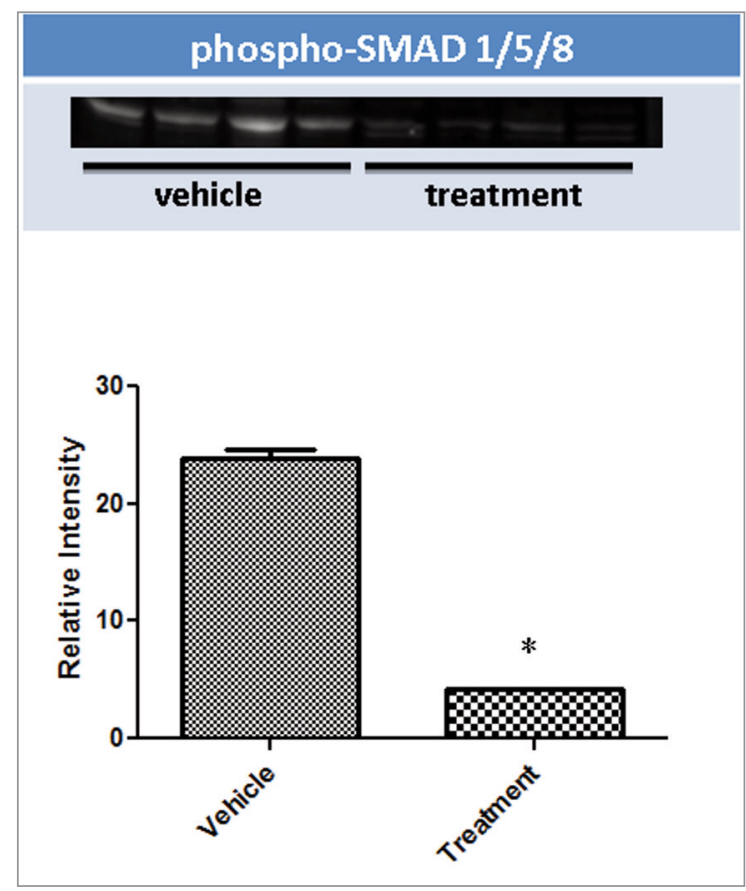

Figure 3. Typical images and band intensity quantification of Western-blot of phosphor-Smad1/5/8 in supraspinatus muscles after RCT with 6 weeks treatment of LDN193189 or vehicle. ${ }^{*}, p<0.05$ compared to vehicle treated control group).

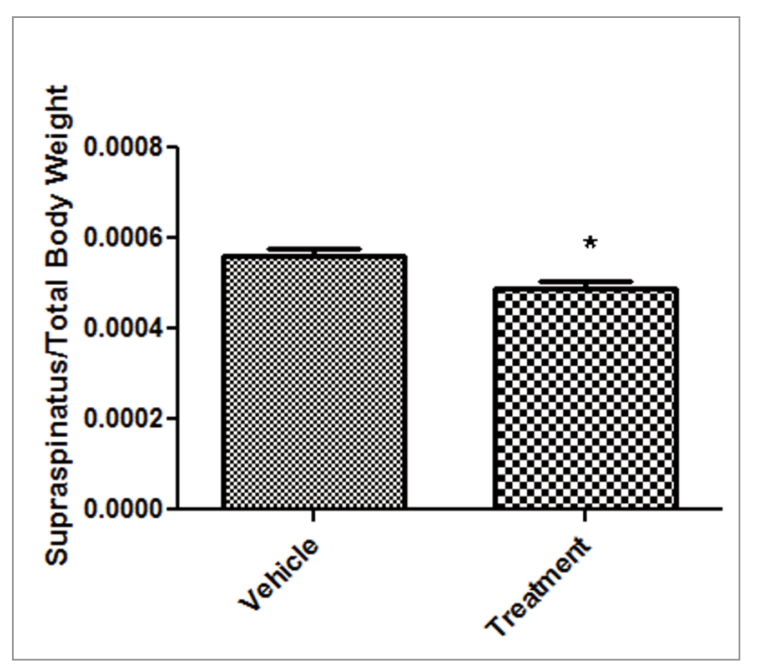

Figure 4. Six weeks treatment of LDN193189 induced reduction of relative weight of supraspinatus muscle (normalized to body weight). ( ${ }^{*}, \mathrm{p}<0.05$ compared to vehicle treated control group). 


\section{Inhibiting BMP signaling reduces supraspinatus muscle fatty infiltration}

Oil-red O staining showed that LDN 193189 treatment significantly reduced fatty infiltration in supraspinatus muscles after RCT compared to vehicle-treated controls (Fig. 6). The average relative fat area percentage (fat area/muscle cross section area $\times 100 \%$ ) reduced 6 fold in the LDN 193189 treated group compared to vehicle treated control group $(p=0.009)$. Western-blot showed that the expression level of SREBP-1, a critical transcription factor in adipogenesis, was significantly decreased by LDN 193189 in supraspinatus muscles after RCT (Fig. 7). These data demonstrated that inhibition BMP pathway significantly reduced fatty infiltration in supraspinatus muscle after RCT.

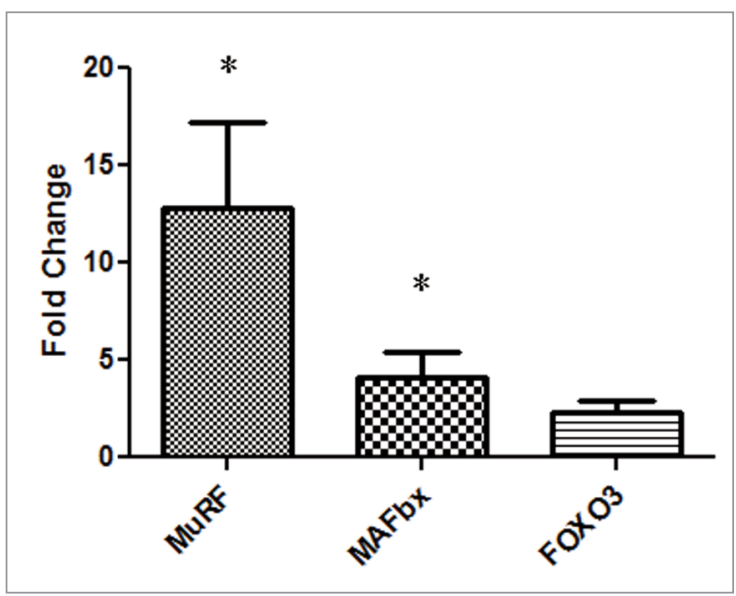

Figure 5. Six weeks treatment of LDN193189 causes increased expression of MuRF-1 and MAFbx in supraspinatus muscle $\left({ }^{*}, p<0.05\right.$ compared to vehicle treated control group).

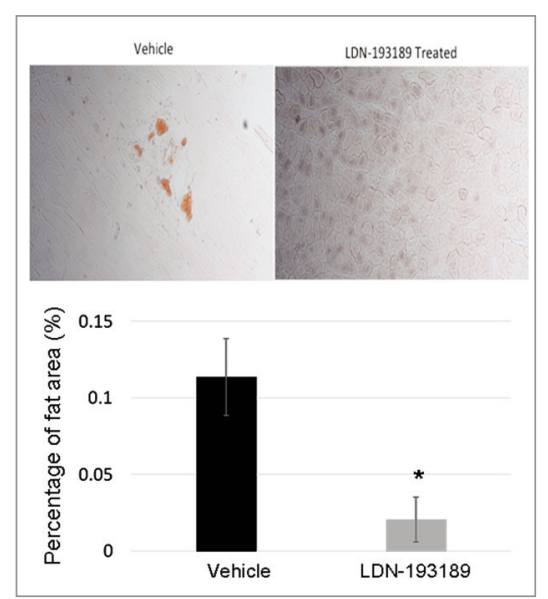

Figure 6. Typical images of Oil-red $O$ for supraspinatus muscles after RCT with 6 weeks treatment of LDN193189 or vehicle. LDN193189 significantly reduced fat deposition in muscle $\left({ }^{*}, p<0.05\right)$

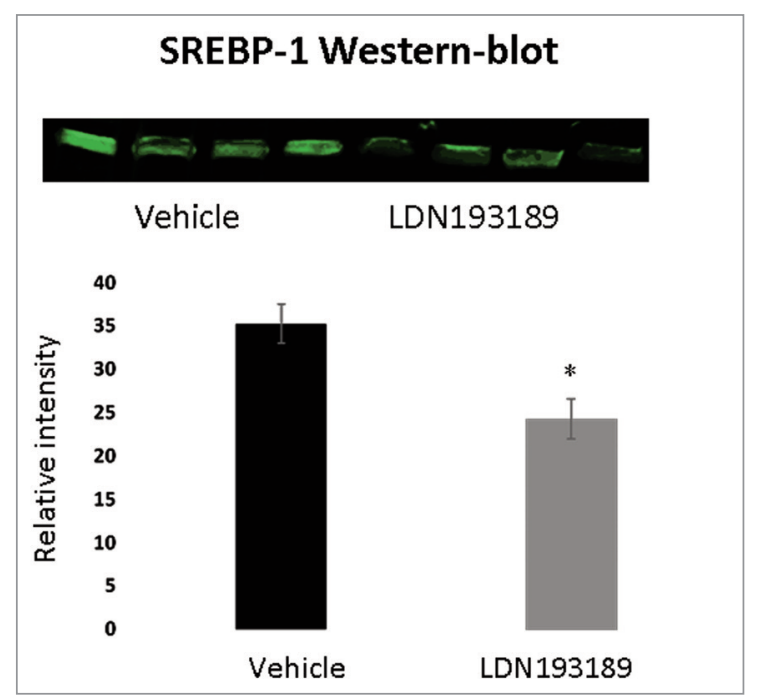

Figure 7. Typical images and band intensity quantification of Western-blot of SREBP-1 in supraspinatus muscles after RCT with 6 weeks treatment of LDN193189 or vehicle. $\left({ }^{*}, p<0.05\right.$ compared to vehicle treated control group).

\section{Discussion}

The purpose of this study was to evaluate the role of the BMP signaling pathway in rotator cuff muscle atrophy and fatty infiltration after massive rotator cuff tears. Our results suggest that BMP signaling plays an important role in rotator cuff muscle atrophy and fatty infiltration after massive RCT.

BMP is well known for its role in bone development. Thus, most previous work on muscle BMP signaling focuses on heterotopic ossification (abnormal bone development in soft tissue). In our previous work in trauma ${ }^{14}$ and spinal cord injury ${ }^{15}$ induced heterotopic ossifications, we found that BMP-2, 4, 7 and 9 are upregulated in muscle during heterotopic ossification.

BMP signaling in muscle atrophy and fatty infiltration is poorly understood. Recently, Sartori et al. demonstrated that BMP-14 has an important role in denervation-induced hind limb muscle atrophy in mice ${ }^{8}$. BMP-14 gene expression was up-regulated over 300 fold as early as 3 days after denervation and remained persistently upregulated. Knocking out BMP14 gene resulted in increased muscle atrophy after denervation. Winbanks et al. also showed that muscle hypertrophy can be induced with over expression of BMP- $7^{16}$. Multiple previous studies also demonstrated that BMP-7 has an important role in regulating stem cell adipogenesis ${ }^{17,18}$. Interestingly, among the BMP ligands panel we tested, we only observed significantly increased BMP-7 and BMP-14 in rotator cuff muscle after RCT in this study. This data implies that BMP-7 and -14 may play a specular role in rotator cuff muscle atrophy and fatty infiltration. Future work is needed to identify the role of BMP-7 and BMP-14 in rotator cuff muscle pathophysiology after massive RCT. 
BMP signaling activate downstream effectors through both the Smad1/5/8 mediated canonical pathway and Smad1/5/8 independent non-canonical pathways. In this study, we blocked BMP signaling using LDN193189, a small molecular BMP type I receptor inhibitor. Significantly reduced phosphorylated Smad1/5/8 expression afterLDN193189 treatment showed that this inhibitor successfully blocked canonical BMP pathway in rotator cuff muscle in our rat model. LDN193189 treatment caused increased muscle atrophy and decreased fatty infiltration in rotator cuff muscles, suggesting BMP signaling may contribute to rotator cuff atrophy and fatty infiltration through the Smad1/5/8 mediated canonical pathway. In spite of reducing fatty infiltration, non-selective blocking canonical BMP signaling with LDN193189 exaggerates rotator cuff atrophy. Thus, pan-BMP inhibitors, LDN193189 may not be optimal treatment option for rotator cuff atrophy and fatty infiltration after RCT. Future works are needed to dissect fine mechanisms responsible for rotator cuff muscle atrophy and fatty infiltration respectively.

Previous works, including our own work in different animal models have shown rotator muscle structure and component changes after tendon injury. A recent study from human muscle biopsy showed increased muscle stiffness after rotator cuff tendon injury ${ }^{19}$. Biopsies of the supraspinatus muscle from full-thickness and massive rotator cuff tear showed upregulation of fibrotic and adipogenic genes ${ }^{20}$. A previous work on rat rotator cuff muscle showed increased miRNA expression and autophagy activity following massive tendon tears ${ }^{21}$. In our recent work, we have discovered significantly upregulated TGF beta signaling in rotator cuff muscle after tendon tears. In this study, we are reporting the role of BMP signaling in rotator cuff muscle atrophy and fatty infiltration. Discovery of novel pathways in rotator cuff muscle pathophysiology may lead to lead to new treatment for this disorder in the future.

There are several limitations in this study. First, BMPs signaling also has been reported to act through Smad independent non-canonical pathways, including p38 MAPK and Akt/mTOR ${ }^{16}$. In this study, we only investigated canonical BMP signaling pathway in rotator cuff muscle atrophy and fatty infiltration. Noncanonical BMP signal pathway was not examined in this study. Second, this experiment was concluded at 6 weeks after RCT. We did not conduct long term follow-up for the BMP inhibitor treatment. Third, we only evaluated BMPs and genes expression using RTPCR. Gene expression change may not proportionally reflex the protein expression function changes of those molecules. The last but not least, though LDN193189 is a commonly used selective BMP inhibitor, it can also inhibits other kinases ${ }^{22}$. The possible "off-target" effect of LDN193189 should be considered. BMP knockout mice may be used to verify the results in this study in the future.

In summary, we observed significantly increased canonical BMP signaling in rotator cuff muscles after $\mathrm{RCT}$ in this study. Inhibiting canonical BMP signaling results in increased muscle atrophy but reduced muscle fatty infiltration after RCT. Future works are needed to distinguish the possible different role of BMP signaling in rotator cuff muscle atrophy and fatty infiltration after RCT.

\section{Acknowledgements}

Funding for this study was provided by $\mathrm{NIH} \mathrm{R03-}$ AR060871, VA RR\&D Merit Review grant RX000195 and Orthopaedic Research Education Foundation. No Authors have any conflicts of interest to disclose.

\section{References}

1. Colvin AC, Egorova N, Harrison AK, Moskowitz A, Flatow EL. National trends in rotator cuff repair. J Bone Joint Surg Am. 2012;94:227-233.

2. Gladstone JN, Bishop JY, Lo IK, Flatow EL. Fatty infiltration and atrophy of the rotator cuff do not improve after rotator cuff repair and correlate with poor functional outcome. Am J Sports Med. 2007;35:719-728.

3. Melis B, Nemoz C, Walch G. Muscle fatty infiltration in rotator cuff tears: descriptive analysis of 1688 cases. Orthopaedics \& traumatology, surgery \& research. OTSR. 2009;95:319-324.

4. Melis B, DeFranco MJ, Chuinard C, Walch G. Natural history of fatty infiltration and atrophy of the supraspinatus muscle in rotator cuff tears. Clin Orthop Relat Res. 2010;468:1498-1505.

5. Melis B, Wall B, Walch G. Natural history of infraspinatus fatty infiltration in rotator cuff tears. J Shoulder Elbow Surg. 2010;19:757-763.

6. Chung SW, Kim JY, Kim MH, Kim SH, Oh JH. Arthroscopic repair of massive rotator cuff tears: outcome and analysis of factors associated with healing failure or poor postoperative function. Am J Sports Med. 2013;41:1674-1683.

7. Winbanks CE, Chen JL, Qian H, et al. The bone morphogenetic protein axis is a positive regulator of skeletal muscle mass. J Cell Biol. 2013;203:345-357.

8. Sartori R, Schirwis E, Blaauw B, et al. BMP signaling controls muscle mass. Nat Genet. 2013;45:1309-1318.

9. Padulo J, Oliva F, Frizziero A, Maffulli N. Muscles, Ligaments and Tendons Journal. Basic principles and recommendations in clinical and fiel science research. MLTJ. 2013;4:250-252.

10. Liu X, Manzano G, Kim HT, Feeley BT. A rat model of massive rotator cuff tears. J Orthop Res. 2011;29:588-595.

11. Joshi SK, Liu X, Samagh SP, et al. mTOR regulates fatty infiltration through SREBP-1 and PPARgamma after a combined massive rotator cuff tear and suprascapular nerve injury in rats. J Orthop Res. 2013;31:724-730.

12. Liu X, Joshi SK, Ravishankar B, Kim HT, Feeley BT. Upregulation of transforming growth factor-beta signaling in a rat model of rotator cuff tears. J Shoulder Elbow Surg. 2014.

13. Joshi SK, Kim HT, Feeley BT, Liu X. Differential ubiquitin-proteasome and autophagy signaling following rotator cuff tears and suprascapular nerve injury. J Orthop Res. 2014;32:138-144.

14. Liu $X$, Kang $H$, Shahnazari M, et al. A novel mouse model of trauma induced heterotopic ossification. J Orthop Res. 2014;32:183-188.

15. Kang H, Dang AB, Joshi SK, et al. Novel mouse model of spinal cord injury-induced heterotopic ossification. J Rehabil Res Dev. 2014;51:1109-1118.

16. Winbanks CE, Chen JL, Qian H, et al. The bone morphogenetic protein axis is a positive regulator of skeletal muscle mass. J Cell Biol. 2013;203:345-357. 
17. Neumann K, Endres M, Ringe J, et al. BMP7 promotes adipogenic but not osteo-/chondrogenic differentiation of adult human bone marrow-derived stem cells in high-density micromass culture. J Cell Biochem. 2007;102:626-637.

18. Zhang $X$, Zhang J, Bauer A, et al. Fine-tuning BMP7 signalling in adipogenesis by UBE2O/E2-230K-mediated monoubiquitination of SMAD6. EMBO J. 2013;32:996-1007.

19. Silldorff MD, Choo AD, Choi AJ, et al. Effect of supraspinatus tendon injury on supraspinatus and infraspinatus muscle passive tension and associated biochemistry. J Bone Joint Surg
Am. 2014:96:e175.

20. Choo A, McCarthy M, Pichika R, et al. Muscle gene expression patterns in human rotator cuff pathology. J Bone Joint Surg Am. 2014;96:1558-1565.

21. Gumucio JP, Davis ME, Bradley JR, et al. Rotator cuff tear reduces muscle fiber specific force production and induces macrophage accumulation and autophagy. J Orthop Res. 2012;30:1963-1970.

22. Sanvitale CE, Kerr G, Chaikuad A, et al. A new class of small molecule inhibitor of BMP signaling. PLoS One. 2013;8:e62721. 